\title{
Transcatheter aortic valve implantation - what the general physician needs to know
}

\author{
Authors: Neil Ruparelia ${ }^{A}$ and Bernard D Prendergast ${ }^{B}$
}

\begin{abstract}
With an increasingly elderly population, the incidence of aortic stenosis (AS) is rising. While surgical aortic valve replacement remains the gold standard treatment for patients with severe symptomatic AS, transcatheter aortic valve implantation (TAVI) has emerged as the treatment of choice for patients who are inoperable or high surgical risk. TAVI has been shown to be associated with a clear mortality benefit when compared with medical therapy and to be at least as good as surgical aortic valve replacement in this patient group. The last few years have seen rapid development in this revolutionary technology in conjunction with increasing centre and operator experience, and indications for the procedure are swiftly expanding. In this review, we summarise the current evidence base and discuss factors that need to be considered by the general physician when contemplating TAVI as a treatment option, including practical aspects, emerging indications and future directions.
\end{abstract}

KEYWORDS: Aortic stenosis, transcatheter aortic valve implantation, TAVI, aortic valve replacement

\section{Introduction}

With an increasingly elderly population, general physicians commonly encounter patients with aortic stenosis (AS), which has a prevalence of $4.6 \%$ in those aged greater than 75 years. ${ }^{1}$ The majority of patients are asymptomatic and the diagnosis of AS is often an incidental finding (usually in the setting of another medical condition). In these individuals, AS is associated with low mortality. ${ }^{2}$ However, the onset of symptoms in severe AS is associated with a bleak prognosis and 50\% mortality within two years without definitive treatment. ${ }^{3}$

The gold standard treatment for severe AS remains surgical aortic valve replacement (SAVR), which is associated with excellent outcomes in contemporary practice. ${ }^{4}$ However, a significant minority of patients presenting to the medical team have multiple comorbidities which are often compounded by significant frailty. In these patients, SAVR is associated

Authors: Ainterventional cardiology fellow, Hammersmith Hospital, Imperial College, London, UK, and Oxford Heart Centre, John

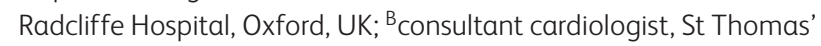
Hospital, London, UK with a $5-10 \%$ risk of major adverse perioperative events and a mortality of up to $20 \%$ at one year. ${ }^{5}$ Given this prohibitively high surgical risk, these patients have historically refused or been denied surgery with no alternative treatment option. Following the first successful human implantation in 2002, ${ }^{6}$ transcatheter aortic valve implantation (TAVI) has rapidly developed over the past few years and is now the established treatment option for inoperable or high-risk surgical patients with severe symptomatic AS. ${ }^{7}$ In this article, we will summarise the current evidence base and discuss factors that need to be taken into account by the general physician when considering TAVI as a treatment option, including practical aspects and future directions.

\section{Current evidence}

Following the first successful TAVI in $2002,{ }^{6}$ the feasibility, efficacy and safety of the procedure were subsequently confirmed in a number of single- and multi-centre registries. ${ }^{8}$

The Placement of AoRTic TraNscathetER Valves (PARTNER) trials were the first prospective randomised studies to compare outcomes of TAVI using the balloon-expandable (BE) Edwards Sapien valve (Edwards LifeSciences, Irvine, CA, USA) with medical management in inoperable patients ${ }^{9}$ (cohort B) and with SAVR in patients of high surgical risk (cohort A). ${ }^{10}$ When compared with medical therapy (plus balloon aortic valvuloplasty as required), TAVI resulted in a significant reduction in one-year mortality (30.7 vs $50.1 \%$ ) that was maintained at three-year follow-up (54.1 vs $80.9 \%) .{ }^{11}$ When TAVI was compared with SAVR in high-risk individuals, all-cause mortality was slightly lower with TAVI at 30 days (3.4 vs $6.5 \%, \mathrm{p}=0.07$ ) but comparable three years following implantation ( 44.2 vs $44 \%$ ) with long-term symptomatic improvement sustained in both groups. ${ }^{12}$

Of late, results of two more contemporary TAVI trials were reported. The non-randomised CoreValve US extremerisk pivotal trial described outcomes of TAVI using the self-expandable (SE) Medtronic CoreValve (Medtronic Inc., Minneapolis, MN, USA) in inoperable patients and demonstrated the efficacy of this treatment option with a $40 \%$ reduction in the one-year combined endpoint of all-cause mortality and stroke. ${ }^{13}$ The randomised CoreValve US high-risk pivotal trial compared the same device with SAVR in patients deemed to be of high surgical risk. For the first time, TAVI was shown to be associated with a mortality benefit ( 14.2 vs $19.1 \%$; $\mathrm{p}<0.0001$ for non-inferiority and $\mathrm{p}=0.04$ for superiority). 
Table 1. Current ESC guidelines for TAVI.

Recommendations

TAVI should only be undertaken by a multidisciplinary 'heart team' including cardiologists and cardiac surgeons and other specialists if necessary.

TAVI should only be performed in hospitals with cardiac surgery onsite.

TAVI is indicated in patients with severe AS who are not suitable for SAVR as assessed by a 'heart team' and who are likely to gain improvement in their quality of life and to have a life expectancy of $>1$ year after consideration of their comorbidities.

TAVI should be considered in high-risk patients IIa

B with severe symptomatic AS who may still be suitable for surgery but in whom TAVI is favoured by a 'heart team' based on the patient's individual risk profile and anatomic suitability.

AS = aortic stenosis; $E S C=$ European Society of Cardiology; SAVR = surgical aortic valve replacement; TAVI = transcatheter aortic valve implantation.

\section{Current guidelines}

Based on current trial and registry data, recommendations by both the European Society of Cardiology (ESC) and the American Heart Association/American College of Cardiology support the role of TAVI in the treatment of severe symptomatic AS in patients deemed inoperable or of high surgical risk, and reaffirms the role of SAVR in all other patients. ${ }^{7,14}$ Current ESC guidelines are summarised in Table 1.

It is important to emphasise that surgical risk is an important factor when deciding which treatment strategy is most suitable for an individual patient. Current guidelines advocate use of the EuroScore ${ }^{15}$ or Society of Thoracic Surgeons ${ }^{16}$ (STS) score $(>20 \%$ or $>10 \%$, respectively, indicate that a patient is of high surgical risk). However, these scores have limitations and may not truly represent clinical status by omitting factors such as frailty that predict adverse outcomes. ${ }^{17}$ A number of frailty scores developed by specialist geratologists (eg McArthur study of successful ageing) are now being evaluated in conjunction with more established surgical risk scores to determine patient risk.
Central to the decision-making process in determining the optimal management strategy is the multidisciplinary 'heart team', comprised of interventional cardiologists, imaging cardiologists, cardiothoracic surgeons, cardiac anaesthetists and general physicians, who use information from clinical assessment, diagnostic work-up (see below) and their collective expertise to guide optimal treatment selection, procedural planning and post-operative care. ${ }^{18}$

\section{Patient work-up}

It is essential that all relevant information is obtained prior to deciding the best treatment strategy for an individual patient. Investigations can be carried out by the referring team or at the TAVI centre although results must be available to inform the heart team.

\section{Confirmation of aortic stenosis}

In the first instance, it is vitally important to confirm the baseline diagnosis of severe AS and be confident that this is the cause of the presenting symptoms. Transthoracic echocardiography and transoesophageal echocardiography are most commonly used to confirm the presence of AS and its severity - current echocardiographic criteria are summarised in Table 2. Other imaging modalities, including computed tomography (CT) and magnetic resonance imaging (MRI) may have a supplementary role.

\section{Anatomical assessment}

Echocardiography and CT are routinely used in a complementary fashion for further anatomical assessment. In addition to the functional assessment of aortic stenosis, information concerning valve anatomy (presence of bicuspid valve), annular size, presence and distribution of valve calcium, relationship of the aortic valve to the left ventricular outflow tract and coronary ostia is essential in determining suitability for TAVI and device selection (Fig 1). Furthermore, assessment of ventricular function and exclusion of intracardiac thrombi are essential for procedural planning and risk assessment.

\section{Access site}

Transfemoral access has a number of advantages, including shorter procedure times, ability to use sedation (and not

Table 2. Echocardiographic diagnostic criteria for severe aortic stenosis.

\begin{tabular}{|c|c|c|}
\hline Echocardiographic criterion & Method of measurement & Limitations \\
\hline Peak jet velocity $>4 \mathrm{~m} / \mathrm{sec}$ & CW Doppler through aortic valve & Doppler measurement must be aligned with transvalve flow \\
\hline Mean gradient $>40 \mathrm{mmHg}$ & AV CW using the Bernoulli formula & Doppler measurement must be aligned with transvalve flow \\
\hline Aortic valve planimetry & Direct measurement of the valve orifice & $\begin{array}{l}\text { Largely subjective, more difficult in the presence of } \\
\text { calcification, poor reproducibility }\end{array}$ \\
\hline AVA $<1 \mathrm{~cm}^{2}$ & $\begin{array}{l}\text { Need to measure: LVOT area, AV VTI } \\
\text { (CW) and LVOT gradient (PW) }\end{array}$ & $\begin{array}{l}\text { Must ensure anatomical measurements are correct to avoid } \\
\text { large errors }\end{array}$ \\
\hline AVA index $<0.6 \mathrm{~cm}^{2} / \mathrm{m}^{2}$ & AVA/BSA & May overdiagnose severe AS in large individuals \\
\hline
\end{tabular}


Fig 1. CT of aortic annulus and arterial tree prior to TAVI. (a) Three-dimensional reconstruction of vasculature, demonstrating calcification (arrows) and tortuosity of peripheral vessels. (b) Twodimensional assessment demonstrating calcification (white arrows) of trileaflet aortic valve, (c) position of right and (d) left coronary ostia. (e) Visualisation of aortic annulus (white line) used in annular sizing and device selection. $\mathrm{CT}=$ computed tomography; TAVI = transcatheter aortic valve implantation.
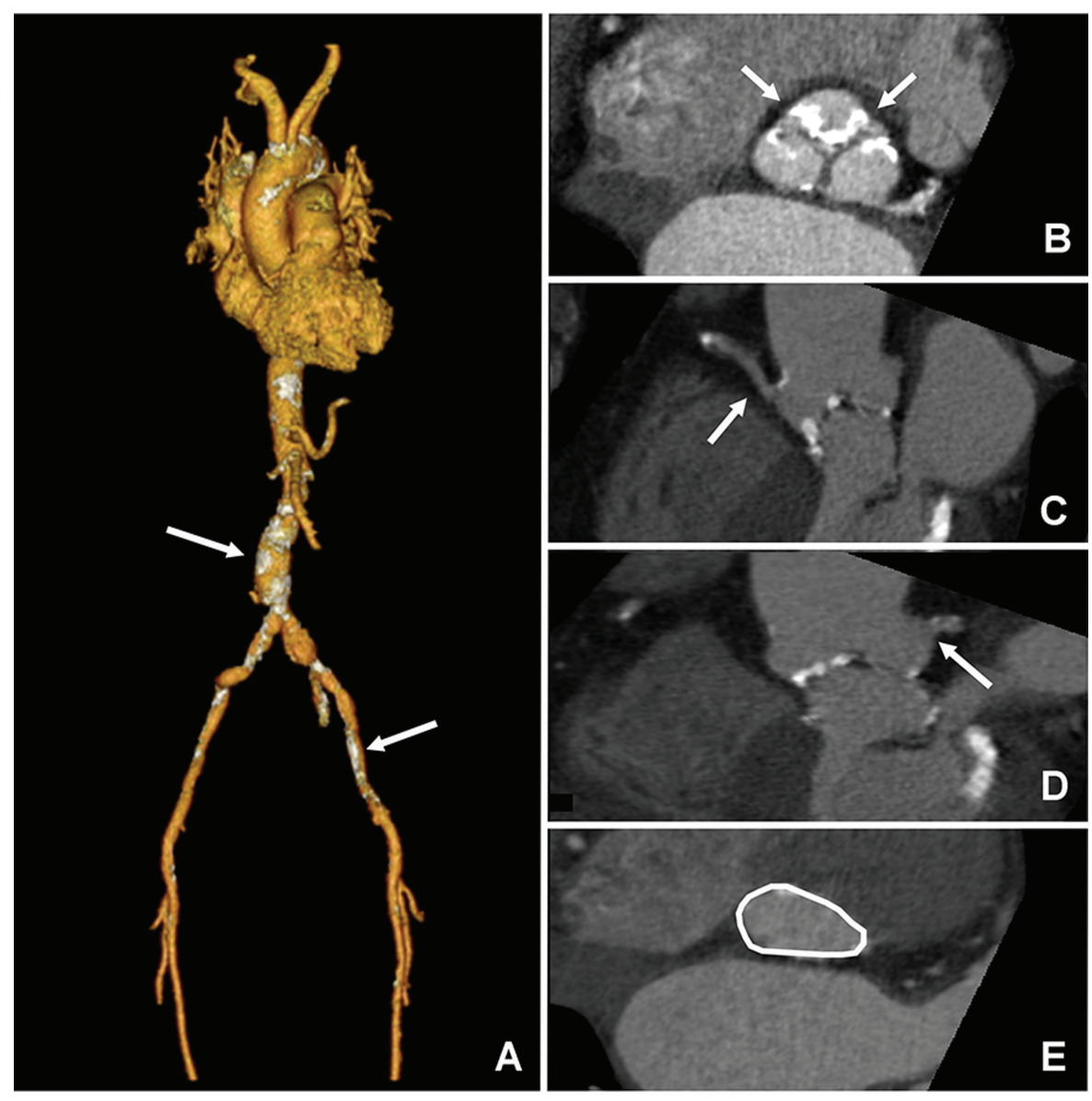

general anaesthesia) and shorter recovery times, and is now the default route for TAVI. However, not all patients are suitable for this approach in view of the high prevalence of peripheral vascular disease in elderly patients with AS. CT and invasive angiography are both used to assess the peripheral vasculature (Fig 1A); current devices require a minimal luminal diameter $>5.5 \mathrm{~mm}$ and the absence of significant calcification and tortuosity.

\section{Coronary angiography}

Patients routinely undergo coronary angiography to evaluate the presence and significance of concomitant coronary artery disease. While coronary artery bypass grafting is routinely carried out for significant coronary artery disease in the setting of SAVR, the benefit of this approach in the setting of TAVI is currently unclear. ${ }^{19}$ Current ongoing trials will provide evidence concerning the best treatment strategy in this patient group.

\section{Blood tests}

Baseline blood investigations should be carried out in all patients to exclude haematological disorders (eg anaemia, clotting abnormalities) and evaluate baseline renal and hepatic function.

\section{Other investigations}

Other routine investigations include pulmonary function tests (for anaesthetic assessment) and carotid ultrasound to determine stroke risk.

\section{The procedure}

Following heart team discussion and patient consent, patients are routinely admitted to hospital the day before their procedure to allow time to meet the medical, surgical and anaesthetic teams and ensure that there are no new medical issues that have arisen since initial assessment. Current guidelines strongly recommend that all TAVI procedures are performed with onsite cardiothoracic surgical support. ${ }^{7}$

\section{Access site and anaesthesia}

Use of the transfemoral route enables the procedure to be carried out under local anaesthesia/sedation in a cardiac catheterisation laboratory (although general anaesthesia is still frequently used in many centres). With development of vascular closure devices, this approach has now become completely percutaneous and no longer requires routine surgical 'cut-down' to access the femoral artery. Other more invasive vascular access sites (eg transapical, transaxillary and 
transaortic) require surgical expertise and general anaesthesia, and may be carried out in a hybrid operating theatre with radiological screening facilities or a cardiac catheterisation laboratory with appropriate specification to ensure procedural sterility.

\section{Device selection}

The majority of worldwide experience to date has been with the BE Edwards valves and SE Medtronic valves (Fig 2). In recent years, a number of advances in valve and delivery system design have significantly reduced complication rates (eg paravalvular leak, vascular injury and bleeding). Newer designs in early commercial phase also incorporate the ability to reposition or retrieve the valve if initial positioning is suboptimal.

All TAVI devices are deployed under fluoroscopic guidance (Fig 2) with frequent use of adjunctive echocardiography. Positioning of $\mathrm{BE}$ valves requires rapid ventricular pacing via a right ventricular temporary pacing wire to reduce aortic pressure and achieve cardiac standstill. This step is usually unnecessary for SE valves. Initial balloon aortic valvuloplasty is no longer routine, although post-dilatation may be required to optimise the result if there is significant paravalvular regurgitation following initial valve deployment.

\section{Recovery and follow-up}

Following the TAVI procedure, patients are usually admitted to a critical care ward for close haemodynamic monitoring and nursing care. According to the site of vascular access, mode of anaesthesia and overall clinical recovery, patients are then 'stepped-down' to a general ward to continue their recovery prior to discharge after 2-3 days. A pre-discharge transthoracic echocardiogram and echocardiography are mandatory to assess prosthetic valve function and exclude atrial fibrillation or new heart block, respectively.

The first clinical follow-up is usually undertaken by the TAVI team at $6-8$ weeks to allow review of symptoms and clinical status, physical examination and echocardiography to assess valve function. In the absence of complications, annual followup thereafter by the local cardiology team is usually sufficient for patient convenience, providing that there is ready access to the TAVI team should problems arise.

During follow-up consultations, physicians should always be vigilant for signs and symptoms of valve failure. While acute causes (eg thrombosis or endocarditis) would warrant emergency transfer to the TAVI team for further management, the development of late prosthesis dysfunction requires medical therapy (eg initiation of diuretics for the management of heart failure) in the first instance and referral for evaluation of further treatment options. Patients more commonly present with general problems including newonset atrial fibrillation or the development of heart failure requiring optimisation of medical therapy (eg antiplatelet therapy, anticoagulation and diuretics). Bearing in mind that the majority of patients that have been treated with TAVI suffer from many comorbidities, it is vitally important that secondary care teams work closely with medical and nursing teams in the community to ensure the best possible management of these complex patients.

\section{Current limitations}

In spite of increasing operator experience and rapid advances in valve and delivery system design, there are a number of current issues that remain the focus of ongoing research to further improve clinical and procedural outcomes in this high-risk patient group.

\section{Paravalvular leak}

Registry and clinical trial data (eg the PARTNER trial ${ }^{12}$ ) have consistently identified moderate and severe paravalvular leak (PVL) as an independent predictor of early and late mortality following TAVI. ${ }^{8,12}$ PVL may arise as a result of poor apposition of the prosthesis with the aortic annulus due to the presence of calcium, an elliptically shaped annulus or undersized device, and appears to affect all devices (to a greater or lesser extent) irrespective of their mode of delivery. Detailed preprocedural imaging (eg the more frequent use of CT) and advances in prosthesis design ensure that PVL is now less frequent. ${ }^{20}$ The impact of these improvements on long-term clinical outcome is under assessment.

\section{Conduction abnormalities}

Conduction disturbances following TAVI are common (BE valves 7-18\%, SE valves 30-83\%). ${ }^{21}$ While permanent pacemaker implantation does not appear to adversely affect short or intermediate clinical outcomes, ${ }^{22}$ longer-term studies are required to assess the true impact of conduction abnormalities.

\section{Stroke}

Although only $2-4 \%$ of all patients suffer clinical signs or symptoms in keeping with a diagnosis of stroke within one year of their procedure, up to $80 \%$ of patients have evidence of cerebral injury when assessed by cerebral MRI following TAVI. ${ }^{23}$ A number of strategies are under investigation in an attempt to reduce the frequency of this important complication, including optimised antiplatelet or anticoagulant therapy, use of cerebral embolic protection devices, and reduced rates of preand post-deployment balloon valvuloplasty.

\section{Cost}

While the high cost of TAVI and need for accumulation of evidence to support safety and efficacy have appropriately limited widespread uptake, initial evidence from the PARTNER $\mathrm{B}$ trial demonstrated that initial higher costs of TAVI during index hospitalisation were more than offset by reduction in overall costs over the first year of follow-up. This has been attributed to a reduction in the need for repeat hospitalisation. In a more recent analysis, TAVI was more cost effective than SAVR in high-risk patients, particularly when performed via the less invasive transfemoral approach. ${ }^{24}$

\section{Expanding indications}

We have focused this manuscript on the treatment of patients with native AS, who form the majority of those considered for TAVI. However, indications have recently expanded 


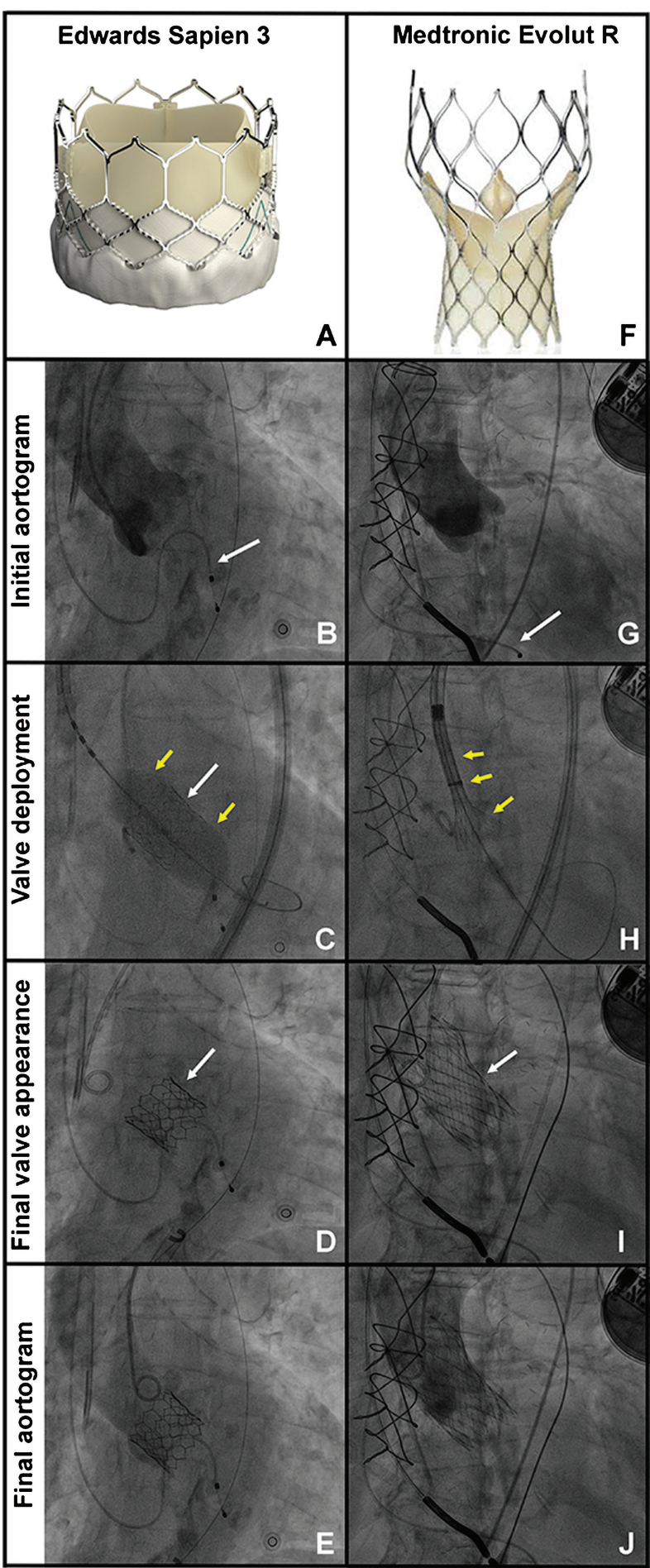

Fig 2. Implantation of balloon-expandable Edwards Sapien 3 valve and self-expanding Medtronic Evolut R valve. Edwards Sapien 3 valve: (a) appearance of valve; (b) representative patient with initial aortogram and temporary pacing wire in situ (white arrow); (c) valve is deployed under rapid pacing with inflation of balloon (yellow arrows) and deployment of valve (white arrow); (d) final fluoroscopic appearance of implanted valve; (e) final aortogram demonstrating functioning valve with no aortic regurgitation. Medtronic Evolut R valve; (f) appearance of valve; (g) representative patient with initial aortogram and temporary pacing wire in situ (white arrow); (h) valve is deployed without need for rapid pacing, with unsheathing of delivery system and self-expansion of valve (yellow arrows); (i) final fluoroscopic appearance of implanted valve (white arrow); (j) final aortogram demonstrating functioning valve with no aortic regurgitation. significantly to encompass the treatment of bicuspid valves, ${ }^{25}$ aortic regurgitation ${ }^{26}$ and degenerating aortic and mitral bioprostheses. ${ }^{27}$ Long-term results concerning the efficacy of these procedures are awaited before adoption into routine clinical practice.

\section{Future developments}

The last few years have seen significant advances in TAVI technology. Coupled with greater operator experience and improved case selection, this has resulted in reduced rates of periprocedural complications and improved short- and medium-term outcomes. Indications have already expanded from the treatment of AS to other valve pathologies. Until now, use of TAVI has been limited to the treatment of inoperable and high-risk surgical patients, however, long-term outcome data concerning clinical outcomes and valve durability will determine whether TAVI should be used routinely for the treatment of lower risk populations. Ongoing trials, including PARTNER IIA, Surgical Replacement and Transcatheter Aortic Valve Implantation (SURTAVI) ${ }^{28}$ and UK TAVI ${ }^{29}$ will hopefully answer this question. Finally, continued refinement of case selection and device technology, coupled with shorter lengths of stay and falling device costs, will ensure that this revolutionary treatment becomes even more attractive to health funders. ${ }^{30}$

\section{Conclusion}

The prevalence of AS will continue to rise in the increasingly elderly population. TAVI is a revolutionary treatment for patients who previously would have been considered inoperable or high risk for conventional surgery. TAVI is now established for the treatment of these patients, and longer-term data concerning valve durability and clinical outcomes are eagerly awaited to ascertain whether TAVI should be routinely offered to lower risk populations. Outcomes and complication rates are set to improve further with accumulating operator experience and technological advances. Not only is TAVI here to stay, but it has also galvanised a revolution in the management of valvular heart disease, and encouraged a new collaborative approach to the management of complex cardiovascular disease.

\section{Conflicts of interest}

Dr Prendergast has received lecture fees from Edwards Lifesciences.

\section{References}

1 Nkomo VT, Gardin JM, Skelton TN et al. Burden of valvular heart diseases: a population-based study. Lancet 2006;368:1005-11.

2 Pellikka PA, Sarano ME, Nishimura RA et al. Outcome of 622 adults with asymptomatic, hemodynamically significant aortic stenosis during prolonged follow-up. Circulation 2005;111:3290-5.

3 Ross J Jr, Braunwald E. Aortic stenosis. Circulation 1968;38:61-7.

4 Brown JM, O'Brien SM, Wu C et al. Isolated aortic valve replacement in North America comprising 108,687 patients in 10 years: changes in risks, valve types, and outcomes in the Society of Thoracic Surgeons National Database. J Thorac Cardiovasc Surg 2009;137:82-90.

5 Speziale G, Nasso G, Barattoni MC et al. Short-term and longterm results of cardiac surgery in elderly and very elderly patients. J Thorac Cardiovasc Surg 2011;141:725-31,731 e1. 
6 Cribier A, Eltchaninoff H, Bash A et al. Percutaneous transcatheter implantation of an aortic valve prosthesis for calcific aortic stenosis: first human case description. Circulation 2002;106:3006-8.

7 Task Force on the management of ST-segment elevation acute myocardial infarction of the European Society of Cardiology, Steg PG, James SK et al. ESC Guidelines for the management of acute myocardial infarction in patients presenting with ST-segment elevation. Eur Heart J 2012;33:2569-619.

8 Tamburino C, Capodanno D, Ramondo A et al. Incidence and predictors of early and late mortality after transcatheter aortic valve implantation in 663 patients with severe aortic stenosis. Circulation 2011;123:299-308.

9 Leon MB, Smith CR, Mack M et al. Transcatheter aortic-valve implantation for aortic stenosis in patients who cannot undergo surgery. N Engl J Med 2010;363:1597-607.

10 Smith CR, Leon MB, Mack MJ et al. Transcatheter versus surgical aortic-valve replacement in high-risk patients. $N$ Engl J Med 2011;364:2187-98.

11 Kapadia SR, Tuzcu EM, Makkar RR et al. Long-term outcomes of inoperable patients with aortic stenosis randomized to transcatheter aortic valve replacement or standard therapy. Circulation 2014, epub ahead of print.

12 Kodali SK, Williams MR, Smith CR et al. Two-year outcomes after transcatheter or surgical aortic-valve replacement. $N$ Engl J Med 2012;366:1686-95.

13 Popma JJ, Adams DH, Reardon MJ et al. Transcatheter aortic valve replacement using a self-expanding bioprosthesis in patients with severe aortic stenosis at extreme risk for surgery. J Am Coll Cardiol 2014;63:1972-81.

14 Holmes DR Jr, Mack MJ, Kaul S et al. 2012 ACCF/AATS/SCAI/STS expert consensus document on transcatheter aortic valve replacement. J Am Coll Cardiol 2012;59:1200-54.

15 Roques F1, Nashef SA, Michel P; EuroSCORE study group. Risk factors for early mortality after valve surgery in Europe in the 1990s: lessons from the EuroSCORE pilot program. J Heart Valve Dis 2001;10:572-8.

16 Ferguson TB Jr, Dziuban SW Jr, Edwards FH et al. The STS National Database: current changes and challenges for the new millennium. Committee to Establish a National Database in Cardiothoracic Surgery, The Society of Thoracic Surgeons. Ann Thorac Surg 2000;69:680-91.

17 Green P, Woglom AE, Genereux P et al. The impact of frailty status on survival after transcatheter aortic valve replacement in older adults with severe aortic stenosis: a single-center experience. JACC Cardiovasc Interv 2012;5:974-81.

18 Hong SJ, Hong MK, Ko YG et al. Multidisciplinary team approach for identifying potential candidate for transcatheter aortic valve implantation. Yonsei Med J 2014;55:1246-52.
19 Gasparetto V, Fraccaro C, Tarantini G et al. Safety and effectiveness of a selective strategy for coronary artery revascularization before transcatheter aortic valve implantation. Catheter Cardiovasc Interv 2013;81:376-83.

20 Webb J, Gerosa G, Lefevre T et al. Multicenter evaluation of a nextgeneration balloon-expandable transcatheter aortic valve. J Am Coll Cardiol 2014;64:2235-43.

21 Rodes-Cabau J. Transcatheter aortic valve implantation: current and future approaches. Nature Rev Cardiol 2012;9:15-29.

22 Buellesfeld L, Stortecky S, Heg D et al. Impact of permanent pacemaker implantation on clinical outcome among patients undergoing transcatheter aortic valve implantation. J Am Coll Cardiol 2012;60:493-501.

23 Kahlert P, Knipp SC, Schlamann M et al. Silent and apparent cerebral ischemia after percutaneous transfemoral aortic valve implantation: a diffusion-weighted magnetic resonance imaging study. Circulation 2010;121:870-8.

24 Orlando R, Pennant M, Rooney S et al. Cost-effectiveness of transcatheter aortic valve implantation (TAVI) for aortic stenosis in patients who are high risk or contraindicated for surgery: a model-based economic evaluation. Health Technol Assess 2013;17:1-86.

25 Mylotte D, Lefevre T, Sondergaard L et al. Transcatheter aortic valve replacement in bicuspid aortic valve disease. J Am Coll Cardiol 2014;64:2330-9.

26 Roy DA, Schaefer U, Guetta V et al. Transcatheter aortic valve implantation for pure severe native aortic valve regurgitation. J Am Coll Cardiol 2013;61:1577-84.

27 Dvir D, Webb J, Brecker S et al. Transcatheter aortic valve replacement for degenerative bioprosthetic surgical valves: results from the global valve-in-valve registry. Circulation 2012;126:2335-44.

28 Medtronic Cardiovascular. Safety and efficacy study of the Medtronic CoreValve System in the treatment of severe, symptomatic aortic stenosis in intermediate risk subjects who need aortic valve replacement (SURTAVI). Available online at https://clinicaltrials.gov/ct2/ show/NCT01586910 [Accessed 15 May 2015].

29 The United Kingdom Transcatheter Aortic Valve Implantation (UK TAVI) Trial. UK TAVI. Oxford: UK TAVI Trial Office, 2014. Available online at www.uktavi.org [Accessed 15 May 2015].

30 Awad W, Mathur A, Baldock L et al. Comparing post-operative resource consumption following transcatheter aortic valve implantation (TAVI) and conventional aortic valve replacement in the UK. J Med Econ 2014;17:357-64.

Address for correspondence: Dr BD Prendergast, St Thomas' Hospital, Westminster Bridge Road, London SE1 7EH, UK. Email: bernard.prendergast@gstt.nhs.uk 